\title{
Connecting the ambitwistor and the sectorized heterotic strings
}

\author{
Thales Azevedo $^{a}$ and Renann Lipinski Jusinskas ${ }^{b}$ \\ ${ }^{a}$ Department of Physics and Astronomy, Uppsala University, \\ Box 516, 751 20, Uppsala, Sweden \\ ${ }^{b}$ Institute of Physics AS CR, \\ Na Slovance 2, 182 21, Prague, Czech Republic \\ E-mail: thales.azevedo@physics.uu.se, renannlj@fzu.cz
}

ABSTRACT: The sectorized description of the (chiral) heterotic string using pure spinors has been misleadingly viewed as an infinite tension string. One evidence for this fact comes from the tree level 3-point graviton amplitude, which we show to contain the usual Einstein term plus a higher curvature contribution. After reintroducing a dimensionful parameter $\ell$ in the theory, we demonstrate that the heterotic model is in fact two-fold, depending on the choice of the supersymmetric sector, and that the spectrum also contains one massive (open string like) multiplet. By taking the limit $\ell \rightarrow \infty$, we finally show that the ambitwistor string is recovered, reproducing the unexpected heterotic state in Mason and Skinner's RNS description.

KEYwords: Supergravity Models, Superstrings and Heterotic Strings

ARXIV EPRINT: 1707.08840 


\section{Contents}

1 Introduction 1

2 The sectorized model and the dimensionful parameter 3

2.13 -point tree-level amplitude for gravitons 4

2.2 Dimensionful parameter 6

3 Alternative formulation $\quad 8$

$\begin{array}{ll}3.1 \text { Cohomology } & 9\end{array}$

$\begin{array}{ll}3.2 & \text { Light-cone description of the massive states } \\ \end{array}$

4 The $\ell \rightarrow \infty$ limit and the connection to ambitwistor strings $\quad 14$

$\begin{array}{ll}\text { 4.1 The heterotic ambitwistor string in the pure spinor formalism } & 15\end{array}$

$\begin{array}{ll}4.2 & \text { The light-cone analysis of the ambitwistor cohomology } \\ \end{array}$

$\begin{array}{llr}5 & \text { Concluding remarks } & 20\end{array}$

$\begin{array}{ll}\text { A Useful OPE's } & 22\end{array}$

\section{Introduction}

After the discovery of the remarkable CHY formulae by Cachazo, He and Yuan [1], which compute N-point tree-level amplitudes of massless states through an integration over points on the Riemann sphere, a natural question was posed: is there a deeper mathematical framework underpinning them?

It did not take long before an elegant answer to that question was proposed. Mason and Skinner presented in [2] the so-called ambitwistor string theory, consisting of a chiral worldsheet model whose tree-level correlation functions give precisely the CHY formulae. Shortly after, Berkovits introduced a manifestly supersymmetric version of the ambitwistor strings [3], described by a chiral form of the usual pure spinor string. The amplitudes computed using this formalism were later shown to give rise to a supersymmetric version of the CHY amplitudes [4].

In the heterotic case, however, both models are not totally satisfying. When restricted to single-trace contributions, the super-Yang-Mills sector gives the expected result for the amplitudes. On the other hand, the supergravity sector failed to reproduce the usual Einstein term. Moreover it seemed impossible in the pure spinor description to find a supergravity vertex operator with the correct properties. This is related to the fact that Berkovits' proposal for the BRST charge does not contain the particle-like Hamiltonian, $H=-\frac{1}{2} P^{m} P_{m}$, which then fails to encode the expected gauge transformations in terms of BRST exact states. 
This issue did not receive much attention until one of the authors, inspired by the works of Chandía and Vallilo [5, 6], developed a new pure-spinor model, referred to as the sectorized heterotic string in the present paper, in which the BRST charge is modified with respect to the original proposal by Berkovits and the description of supergravity states was made possible [7]. As a consistency check for the new BRST charge, the sectorized string was later coupled to a generic heterotic background [8] and we were able to show that requiring (classical) nilpotency of the BRST charge is equivalent to imposing the classical constraints on the background superfields, which had been previously shown by Berkovits and Howe to imply the supergravity and super Yang-Mills equations of motion [9].

Providing a sensible vertex operator for the states in the supergravity sector was an important achievement of the sectorized model. ${ }^{1}$ One would then be able to use that vertex operator to compute 3-point, tree-level correlation function of gravitons, and hopefully to reproduce the usual amplitude coming from Einstein gravity. However, that is not the case, as we will later show. In fact, the 3-point amplitude includes the usual terms coming from Einstein gravity but it also contains terms of order four in the momenta, which resemble a Gauss-Bonnet theory.

The presence of a higher-order term in the 3-point amplitude motivated us to reintroduce a dimensionful parameter in the theory and then look for massive states. We found that in addition to the massless states the BRST cohomology contains a single massive level, which is reminiscent of the first massive level of the open superstring. We investigated these extra states in detail, as well as their relation to the original heterotic ambitwistor string. In particular, there is a massive 3 -form which reduces in the infinite length (tensionless) limit to the unexpected massless 3-form originally encountered by Mason and Skinner. This can be explicitly shown in an $\mathrm{SO}(8)$-covariant form via a Del Giudice-Di Vecchia-Fubini (DDF) construction of the vertex operator.

In [10], Siegel already observed that ambitwistor strings could be viewed as tensionless string-like models. The connection between the sectorized model and the ambitwistor string as its infinite length limit is similar to the one briefly considered by Casali and Tourkine in [11] for the bosonic string, which in turn is closely related to the work of Huang, Siegel and Yuan in [12]. Indeed, there are many similarities between our results and those obtained by Huang et al. . In particular, the 3-point graviton amplitude we compute in the present paper agrees with their results for the chiral heterotic string, including the two different cases depending on which sector is "flipped". In our case, this corresponds to the two inequivalent ways of sectorizing the string, which we call A and B models. As usual, the pure spinor approach is more covenient in the supersymmetric analysis.

As already mentioned, we give compelling evidence that the tensionless limit of the sectorized string is equivalent to the ambitwistor string. But more surprisingly, our results also seem to be connected to the twistor string theory studied by Berkovits and Witten [13, 14]. In [15] a formula for the heterotic ambitwistor amplitude of $n$ gravitons was found which reduces to that of Berkovits-Witten in the four-dimensional MHV case. We believe this subject deserves more investigation, and expect to address it in future work. ${ }^{2}$

\footnotetext{
${ }^{1}$ More precisely, while the unintegrated vertex operators are known, the integrated ones are still missing in the sectorized models. Although this is not the focus of this work, we briefly discuss this topic in section 5 .

${ }^{2}$ We are thankful to Henrik Johansson for bringing this to our attention.
} 
This paper is organized as follows. In section 2, after reviewing the sectorized model, we present the tree level 3-point graviton amplitude with its different contributions, motivating the introduction of a length parameter $\ell$ and a more thorough study of the cohomology. In section 3 , we propose an alternative definition of the heterotic sectorized string, demonstrating that the physical spectrum contains also one massive (open string like) multiplet. Section 4 is dedicated to the analysis of the $\ell \rightarrow \infty$ limit and a new proposal for the heterotic ambitwistor BRST charge in the pure spinor formalism and its cohomology. We summarize our results and discuss open problems in section 5. Appendix A lists the relevant OPE's used in the paper.

\section{The sectorized model and the dimensionful parameter}

The action for the heterotic sectorized string in the pure spinor formalism is given by

$$
S=\int d^{2} z\left\{P_{m} \bar{\partial} X^{m}+p_{\alpha} \bar{\partial} \theta^{\alpha}+w_{\alpha} \bar{\partial} \lambda^{\alpha}+\bar{b} \bar{\partial} \bar{c}+\mathcal{L}_{C}\right\},
$$

where $\left(X^{m}, \theta^{\alpha}\right)$ denote the target space supercoordinates of the string and $\left(P_{m}, p_{\alpha}\right)$ their conjugate momenta, with $m=0, \ldots, 9$ and $\alpha=1, \ldots, 16 ; \lambda^{\alpha}$ is the pure spinor satisfying $\left(\lambda \gamma^{m} \lambda\right)=0$ and $w_{\alpha}$ its conjugate; $(\bar{b}, \bar{c})$ is the usual Virasoro ghost pair. The gamma matrices, $\gamma_{\alpha \beta}^{m}$ and $\gamma^{m \alpha \beta}$, satisfy $\left\{\gamma^{m}, \gamma^{n}\right\}=2 \eta^{m n}$, where $\eta^{m n}$ is the $\operatorname{SO}(9,1)$ metric. The Lagrangian $\mathcal{L}_{C}$ describes the $\mathrm{SO}(32)$ or $E(8) \times E(8)$ gauge sector with energy-momentum $T_{C}$ and central charge 16 , and the associated current algebra is realized by the (holomorphic) generators $J^{I}$, with $I$ denoting the adjoint representation of the gauge group.

The fundamental feature of this chiral model is its interpretation in terms of two sectors, $(+)$ and (-), that resemble the usual left and right movers of the superstring [7]. There are two decoupled sets of operators, $\mathcal{O}_{ \pm}$, organized $\operatorname{as}^{3}$

$$
\begin{aligned}
& \mathcal{O}_{+}=\left\{P_{m}^{+}, \bar{b}, \bar{c}, J^{I}\right\}, \\
& \mathcal{O}_{-}=\left\{P_{m}^{-}, d_{\alpha}, \theta^{\alpha}, w_{\alpha}, \lambda^{\alpha}\right\},
\end{aligned}
$$

with

$$
\begin{aligned}
P_{m}^{+} & =P_{m}+\partial X_{m} \\
P_{m}^{-} & =P_{m}-\partial X_{m}-\left(\theta \gamma_{m} \partial \theta\right) \\
d_{\alpha} & =p_{\alpha}-\frac{1}{2}\left(\gamma^{m} \theta\right)_{\alpha} P_{m}^{-}-\frac{1}{4}\left(\theta \gamma^{m} \partial \theta\right)\left(\gamma_{m} \theta\right)_{\alpha} .
\end{aligned}
$$

The $\mathcal{N}=1$ supersymmetry of the heterotic string is compatible with the sectorized formulation and all the operators displayed in (2.3) are invariant under the supersymmetry charge

$$
q_{\alpha}=\oint\left\{p_{\alpha}+\frac{1}{2} P_{m}^{-}\left(\gamma^{m} \theta\right)_{\alpha}+\frac{5}{12}\left(\theta \gamma^{m} \partial \theta\right)\left(\gamma_{m} \theta\right)_{\alpha}\right\}
$$

\footnotetext{
${ }^{3}$ There are in fact two inequivalent ways of spliting the worldsheet operators in two decoupled sets, as explained in section 3. Here we review the choice made in previous works [7,8], referred to in the present paper as the A model.
} 
The two sectors have characteristic (pseudo) energy-momentum tensors defined by

$$
\begin{aligned}
& T_{-} \equiv \frac{1}{4} \eta^{m n} P_{m}^{-} P_{n}^{-}-d_{\alpha} \partial \theta^{\alpha}-w_{\alpha} \partial \lambda^{\alpha} \\
& T_{+} \equiv-\frac{1}{4} \eta^{m n} P_{m}^{+} P_{n}^{+}+T_{C}-\bar{b} \partial \bar{c}-\partial(\bar{b} \bar{c})
\end{aligned}
$$

which combine to form the energy-momentum tensor associated to the action (2.1),

$$
\begin{aligned}
T & =T_{+}+T_{-}, \\
& =-P_{m} \partial X^{m}-p_{\alpha} \partial \theta^{\alpha}-w_{\alpha} \partial \lambda^{\alpha}-2 \bar{b} \partial \bar{c}-\bar{c} \partial \bar{b}+T_{C} .
\end{aligned}
$$

The BRST charge of the model is defined to be

$$
Q=\oint\left\{\lambda^{\alpha} d_{\alpha}+\bar{c} T_{+}-\bar{b} \bar{c} \partial \bar{c}\right\}
$$

and naturally incorporates the sector splitting. Furthermore, its cohomology consistently describes the expected heterotic massless spectrum. In addition to the super Yang-Mills states, encoded by the superfield $A_{\alpha}^{I}$ in the vertex operator $U_{\mathrm{SYM}}$,

$$
\begin{aligned}
U_{\mathrm{SYM}} & =\lambda^{\alpha} \bar{c} A_{\alpha}^{I} J_{I}, \\
\gamma_{m n p q r}^{\alpha \beta} D_{\alpha} A_{\beta}^{I} & =0, \\
\delta_{\Sigma} A_{\alpha}^{I} & =D_{\alpha} \Sigma^{I},
\end{aligned}
$$

the cohomology of $Q$ contains also the $\mathcal{N}=1$ supergravity states, encoded by the superfield $A_{\alpha}^{m}$ in the vertex operator $U_{\mathrm{SG}}$,

$$
\begin{aligned}
U_{\mathrm{SG}} & =\lambda^{\alpha} \bar{c} A_{\alpha}^{m} P_{m}^{+}, \\
\gamma_{m n p q r}^{\alpha \beta} D_{\alpha} A_{\beta}^{s} & =0, \\
\partial^{n} \partial_{n} A_{\alpha}^{m}-\partial^{m} \partial_{n} A_{\alpha}^{n} & =0, \\
\delta_{\Sigma} A_{\alpha}^{m} & =D_{\alpha} \Sigma^{m}+\partial^{m} \Sigma_{\alpha} .
\end{aligned}
$$

The superfields $\Sigma^{I}, \Sigma_{\alpha}$ and $\Sigma^{m}$ are the gauge parameters of the transformations $(2.8 \mathrm{c})$ and $(2.9 \mathrm{~d})$. More details can be found in [7].

It is worthwhile to point out that the supergravity sector does not have a clear description in the ambitwistor heterotic string $[2,3]$. Providing a sensible vertex operator for the supergravity states was an important achievement of the sectorized model. Going further, we were also able to show in [8] that the model imposes the correct (on-shell) constraints when coupled to a generic heterotic background. As another test for the sectorized model, we can compute the 3-point, tree-level correlation function of gravitons using the vertex operator (2.9a), as we show in the following subsection.

\subsection{3 -point tree-level amplitude for gravitons}

It is clear that the vertex $(2.9 \mathrm{a})$ has a very similar structure to the ordinary vertex in the full string. Thus, thinking of the sectorized string as an infinite tension limit of the usual 
string, one would expect the 3-point graviton amplitude to give rise to the usual expression coming from Einstein gravity. However, that is not the case, as seen from the computation we now sketch.

The unintegrated vertex operator for the graviton labeled by $j(j \in\{1,2,3\})$ is given by

$$
U_{(j)}=\bar{c} \lambda^{\alpha} A_{(j) \alpha}^{m}(\theta) P_{m}^{+} e^{i k_{(j)} \cdot X},
$$

and $A_{(j) \alpha}^{m}(\theta)$ can be gauged to the following expansion,

$$
A_{(j) \alpha}^{m}(\theta)=\frac{1}{2}\left(\gamma_{n} \theta\right)_{\alpha} \epsilon_{(j)}^{m n}-\frac{i}{16}\left(\gamma^{q} \theta\right)_{\alpha}\left(\theta \gamma_{n p q} \theta\right) k_{(j)}^{n} \epsilon_{(j)}^{m p}+\mathcal{O}\left(\theta^{5}\right),
$$

where $\epsilon_{(j)}^{m n}$ is a symmetric-traceless polarization tensor such that $\eta_{m n} k_{(j)}^{n} \epsilon_{(j)}^{m p}=0$.

The 3-point scattering amplitude at tree level is given by the usual correlator of three vertex operators inserted at arbitrary points $z_{j}$ on the 2 -sphere:

$$
\left\langle U_{(1)}\left(z_{1}\right) U_{(2)}\left(z_{2}\right) U_{(3)}\left(z_{3}\right)\right\rangle=\left\langle\bar{c}\left(z_{1}\right) \bar{c}\left(z_{2}\right) \bar{c}\left(z_{3}\right)\right\rangle\left\langle\lambda^{\alpha} A_{(1) \alpha}^{m}(\theta) \lambda^{\beta} A_{(2) \beta}^{n}(\theta) \lambda^{\gamma} A_{(3) \gamma}^{p}(\theta)\right\rangle C_{X P},
$$

where $C_{X P}$ is the correlator involving $X$ and $P^{+}$and we have separated the independent contributions, to which we now turn.

The $\bar{c}$-ghost correlator is the same as in bosonic string theory and is known to give (up to an overall factor)

$$
\left\langle\bar{c}\left(z_{1}\right) \bar{c}\left(z_{2}\right) \bar{c}\left(z_{3}\right)\right\rangle=z_{12} z_{23} z_{31} \quad\left(z_{i j}:=z_{i}-z_{j}\right) .
$$

The $\lambda$ and $\theta$ zero-mode integration is performed via the usual BRST-invariant measure factor $\left\langle\left(\lambda \gamma^{m} \theta\right)\left(\lambda \gamma^{n} \theta\right)\left(\lambda \gamma^{p} \theta\right)\left(\theta \gamma_{m n p} \theta\right)\right\rangle=1$, such that the second correlator in (2.12) reduces to (again up to normalization)

$$
i\left(k_{(3)} \cdot \epsilon_{(1)}\right)^{m} \eta_{q r} \epsilon_{(2)}^{n q} \epsilon_{(3)}^{p r}+i\left(k_{(1)} \cdot \epsilon_{(2)}\right)^{n} \eta_{q r} \epsilon_{(3)}^{p q} \epsilon_{(1)}^{m r}+i\left(k_{(2)} \cdot \epsilon_{(3)}\right)^{p} \eta_{q r} \epsilon_{(1)}^{m q} \epsilon_{(2)}^{n r} .
$$

Finally, there are two contributions to $C_{X P}$. The first one involves Wick contractions between $X$ and $P^{+}$and also between two $P^{+}$'s (note that $P^{+}$has a nontrivial OPE with itself due to the mixing of $P^{m}$ and $\partial X^{m}$, cf. equation (2.3a)). The second one involves only contractions between $X$ and $P^{+}$. Recall that there are no contractions between the exponentials since the $X X$ OPE is trivial. The first set of contractions gives

$$
\begin{aligned}
2 i\left[z_{12}^{-2} \eta_{m n}\left(z_{31}^{-1} k_{(1) p}+z_{32}^{-1} k_{(2) p}\right)+z_{23}^{-2} \eta_{n p}\left(z_{12}^{-1} k_{(2) m}+z_{13}^{-1} k_{(3) m}\right)\right. & \left.+z_{31}^{-2} \eta_{m p}\left(z_{23}^{-1} k_{(3) n}+z_{21}^{-1} k_{(1) n}\right)\right] \\
=-2 i\left(z_{12} z_{23} z_{31}\right)^{-1}\left(\eta_{m n} k_{(1) p}+\right. & \left.\eta_{n p} k_{(2) m}+\eta_{m p} k_{(3) n}\right)
\end{aligned}
$$

where we have used momentum conservation and transversality of the polarization tensors, for example,

$$
z_{31}^{-1} k_{(1) p}+z_{32}^{-1} k_{(2) p}=\left(z_{31}^{-1}-z_{32}^{-1}\right) k_{(1) p}=\frac{z_{12}}{z_{31} z_{32}} k_{(1) p} .
$$

Combining these results and using the shorthand notation $\epsilon_{(i)}^{m n} \equiv \epsilon_{(i)}^{m} \epsilon_{(i)}^{n}$, the first contribution to the amplitude can be expressed as

$$
\mathcal{M}_{1}=2\left[\left(\epsilon_{(1)} \cdot \epsilon_{(2)}\right)\left(\epsilon_{(3)} \cdot k_{(1)}\right)+\left(\epsilon_{(2)} \cdot \epsilon_{(3)}\right)\left(\epsilon_{(1)} \cdot k_{(2)}\right)+\left(\epsilon_{(3)} \cdot \epsilon_{(1)}\right)\left(\epsilon_{(2)} \cdot k_{(3)}\right)\right]^{2},
$$

just like the usual term coming from Einstein gravity. 
To conclude the computation of this amplitude, we need to find the second contribution in $C_{X P}$. There are two independent ways of contracting the $P^{+}$'s with the $X^{\prime}$ 's in the exponentials, and the result is

$$
i\left(z_{12} z_{23} z_{31}\right)^{-1}\left(k_{(2) m} k_{(3) n} k_{(1) p}-k_{(3) m} k_{(1) n} k_{(2) p}\right) .
$$

Thus the second contribution to the amplitude is given by

$$
\begin{aligned}
\mathcal{M}_{2}=2\left[\left(\epsilon_{(1)} \cdot \epsilon_{(2)}\right)\left(\epsilon_{(3)} \cdot k_{(1)}\right)+\left(\epsilon_{(2)} \cdot \epsilon_{(3)}\right)\left(\epsilon_{(1)}\right.\right. & \left.\left.\cdot k_{(2)}\right)+\left(\epsilon_{(3)} \cdot \epsilon_{(1)}\right)\left(\epsilon_{(2)} \cdot k_{(3)}\right)\right] \times \\
& \times\left(\epsilon_{(1)} \cdot k_{(2)}\right)\left(\epsilon_{(2)} \cdot k_{(3)}\right)\left(\epsilon_{(3)} \cdot k_{(1)}\right),
\end{aligned}
$$

which can be interpreted in terms of a Gauss-Bonnet theory. More precisely, any combination of the curvature-squared terms in a Lagrangian would give rise to (2.19), since the Ricci tensor vanishes on-shell. Nonetheless, the Gauss-Bonnet choice renders the theory ghost-free (see, for example, [16]).

Note that $\mathcal{M}_{1}$ and $\mathcal{M}_{2}$ have different powers in the gravitons' momenta, an unexpected feature of a model thought to be dimensionless. In what follows we will discuss this in detail.

\subsection{Dimensionful parameter}

A more careful look at the model raises some questions about the role played by dimensionality. In this direction, the operators defined in (2.3) lack a dimensionful parameter, which in the usual string would be represented by $\alpha^{\prime}$.

The fundamental observation here is that the sectorized string was originally thought of as an alternative formulation of the ambitwistor string, which in turn is viewed as an infinite tension string $\left(\alpha^{\prime} \rightarrow 0\right)$. In order to avoid confusion, we will introduce a parameter of length dimension denoted by $\ell$ and later on we will discuss its relation to $\alpha^{\prime}$.

To introduce $\ell$ consistently, we will take advantage of a global scaling symmetry of the chiral action (2.1) and rescale the worldsheet fields as

$$
\begin{array}{cccc}
X^{m} \rightarrow \ell^{-1} X^{m}, & \theta^{\alpha} \rightarrow \ell^{-\frac{1}{2}} \theta^{\alpha}, & \lambda^{\alpha} \rightarrow \ell^{-\frac{1}{2}} \lambda^{\alpha}, & \bar{c} \rightarrow \ell^{-2} \bar{c}, \\
P_{m} \rightarrow \ell^{+1} P_{m}, & p_{\alpha} \rightarrow \ell^{+\frac{1}{2}} p_{\alpha}, & w_{\alpha} \rightarrow \ell^{+\frac{1}{2}} w_{\alpha}, & \bar{b} \rightarrow \ell^{+2} \bar{b} .
\end{array}
$$

The Lagrangian of the gauge sector, $\mathcal{L}_{C}$, will not change and $J_{I} \rightarrow J_{I}$. These particular weights were chosen in such a way that the BRST charge (2.7) is finite in the limit $\ell \rightarrow \infty$. Now we are able to dimensionally balance the supersymmetric invariants defined before:

$$
\begin{aligned}
d_{\alpha} & =p_{\alpha}-\frac{1}{2} P_{m}\left(\gamma^{m} \theta\right)_{\alpha}+\frac{1}{2 \ell^{2}} \Pi^{m}\left(\gamma_{m} \theta\right)_{\alpha}, \\
P_{m}^{-} & =P_{m}-\frac{1}{\ell^{2}} \partial X_{m}-\frac{1}{\ell^{2}}\left(\theta \gamma_{m} \partial \theta\right), \\
P_{m}^{+} & =P_{m}+\frac{1}{\ell^{2}} \partial X_{m} . \\
\Pi^{m} & =\partial X^{m}+\frac{1}{2}\left(\theta \gamma^{m} \partial \theta\right), \\
T_{-} & \equiv \frac{1}{4} \eta^{m n} P_{m}^{-} P_{n}^{-}-\frac{1}{\ell^{2}}\left(d_{\alpha} \partial \theta^{\alpha}+w_{\alpha} \partial \lambda^{\alpha}\right), \\
T_{+} & \equiv-\frac{1}{4} \eta^{m n} P_{m}^{+} P_{n}^{+}-\frac{1}{\ell^{2}}\left(2 \bar{b} \partial \bar{c}-\bar{c} \partial \bar{b}-T_{C}\right) .
\end{aligned}
$$


Aside from $\Pi_{m}=\frac{\ell^{2}}{2}\left(P_{m}^{+}-P_{m}^{-}\right)$, the operators above correspond to those displayed at the beginning of this section. Note that the energy-momentum tensor is given by $T=$ $\ell^{2}\left(T_{+}+T_{-}\right)$while the generalized particle like Hamiltonian is $\mathcal{H}=\left(T_{+}-T_{-}\right)$,

$$
\mathcal{H}=-\frac{1}{4} \eta^{m n} P_{m}^{+} P_{n}^{+}-\frac{1}{4} \eta^{m n} P_{m}^{-} P_{n}^{-}+\frac{1}{\ell^{2}}\left[T_{C}-\bar{b} \partial \bar{c}-\partial(\bar{b} \bar{c})+d_{\alpha} \partial \theta^{\alpha}+w_{\alpha} \partial \lambda^{\alpha}\right],
$$

which is supersymmetric and BRST-exact [7].

The BRST charge has the same form as before and can be organized as

$$
\begin{aligned}
Q & =Q_{\lambda}+Q_{+}, \\
Q_{\lambda} & \equiv \oint \lambda^{\alpha} d_{\alpha}, \\
Q_{+} & \equiv \oint \bar{c}\left(T_{+}+\frac{1}{\ell^{2}} \bar{b} \partial \bar{c}\right),
\end{aligned}
$$

cf. the operators displayed in (2.21).

After reintroducing this "hidden" parameter $\ell$ back into the theory, it is reasonable to take a more careful look at the cohomology of (2.23). Considering only eigenstates of momentum, it is possible to show that the massless vertex operator with ghost number two has the general form

$$
U=\partial \bar{c} \lambda^{\alpha} A_{\alpha}+\left(P_{m}^{+}, \bar{c} \lambda^{\alpha} A_{\alpha}^{m}\right)+\bar{c} \lambda^{\alpha} J_{I} A_{\alpha}^{I}+\left(P_{m}^{+}, \bar{c} \partial \bar{c} A^{m}\right)+\lambda^{\alpha} \lambda^{\beta} A_{\alpha \beta}+\frac{1}{\ell^{2}} \bar{c} \partial^{2} \bar{c} A,
$$

with ordering prescription defined as

$$
\left(\mathcal{O}_{1}, \mathcal{O}_{2}\right)(y) \equiv \frac{1}{2 \pi i} \oint \frac{d z}{z-y} \mathcal{O}_{1}(z) \mathcal{O}_{2}(y)
$$

The superfields $A, A_{\alpha}, A^{m}, A_{\alpha}^{m}, A_{\alpha}^{I}$ and $A_{\alpha \beta}$ have gauge transformations given by

$$
\begin{aligned}
\delta A_{\alpha} & =D_{\alpha} \Omega \\
\delta A^{m} & =-\frac{1}{2} \partial^{m} \Omega \\
\delta A_{\alpha \beta} & =\frac{1}{2} D_{\alpha} \Omega_{\beta}+\frac{1}{2} D_{\beta} \Omega_{\alpha} \\
\delta A_{\alpha}^{m} & =\frac{1}{2} \partial^{m} \Omega_{\alpha}-D_{\alpha} \Omega^{m} \\
\delta A_{\alpha}^{I} & =D_{\alpha} \Omega^{I} \\
\delta A & =-\Omega+\frac{1}{2} \partial_{m} \Omega^{m}
\end{aligned}
$$

and BRST-closedness of $U$ implies the followingequations of motion:

$$
\begin{aligned}
\lambda^{\alpha} \lambda^{\beta} D_{\beta} A_{\alpha} & =0, \\
D_{\alpha} A^{m}+\frac{1}{2} \partial^{m} A_{\alpha} & =0 .
\end{aligned}
$$




$$
\begin{aligned}
D_{\alpha} A+A_{\alpha}+\frac{1}{2} \partial_{m} A_{\alpha}^{m} & =0, \\
\frac{1}{2 \ell^{2}} \partial_{m} A^{m} & =0, \\
\lambda^{\alpha} \lambda^{\beta} \lambda^{\gamma} D_{\gamma} A_{\alpha \beta} & =0, \\
\lambda^{\alpha} \lambda^{\beta} D_{\beta} A_{\alpha}^{m}-\frac{1}{2} \lambda^{\alpha} \lambda^{\beta} \partial^{m} A_{\alpha \beta} & =0, \\
\lambda^{\alpha} \lambda^{\beta} D_{\beta} A_{\alpha}^{I} & =0 .
\end{aligned}
$$

The super Maxwell field is a solution of (2.27a). However, equation (2.27b) implies that $A_{\alpha} \neq 0$ and $A^{m} \neq 0$ are pure gauge, cf. (2.26a) and (2.26b). The same argument holds for $A_{\alpha \beta}$. Equation $(2.27 \mathrm{e})$ has a known solution, namely the antifields of super Maxwell [17]. On the other hand, if $A_{\alpha \beta}$ is not zero, equation (2.27f) implies it is pure gauge, cf. equation (2.26c). Therefore $A_{\alpha}=A^{m}=A_{\alpha \beta}=0$. Equation (2.27c) states that the longitudinal part of $A_{\alpha}^{m}$ is also pure gauge, so we can use $\partial_{m} \Omega^{m}$ to fix $A=0$ and make $A_{\alpha}^{m}$ transversal. In the end, we are left with the known massless content given in (2.8) and (2.9), respectively super Yang-Mills and $\mathcal{N}=1$ supergravity. Note that the physical states have ghost number one with respect to each sector, $(+)$ and $(-)$, but never ghost number two within the same sector.

It is now natural to question whether the existence of massive states has been so far ignored. For massive solutions, it is easy to show that the vertex (2.24) is BRST-exact. However, there is one possible vertex construction that was not considered before,

$$
U=\bar{c} U_{-}
$$

where $U_{-}$is a ghost number one, conformal weight +1 operator composed out of superfields combined with currents from the $(-)$ sector. Computing the commutator of $(2.28)$ with the BRST charge, one obtains

$$
[Q, U]=\left(\frac{1}{\ell^{2}}+\frac{\square}{4}\right) \bar{c} \partial \bar{c} U_{-}-\bar{c}\left\{Q_{\lambda}, U_{-}\right\}
$$

The two terms on the right hand side of the above equation are independent and must vanish separately. The vanishing of the first one implies the mass-shell condition $M^{2}=-\frac{4}{\ell^{2}}$, i.e. a tachyon. The vanishing of the second term implies that $U_{-}$is in the cohomology of $Q_{\lambda}$, the usual pure spinor BRST charge. These two conditions are incompatible simply because the cohomology of $Q_{\lambda}$ is supersymmetric, ensuring the absence of tachyonic states.

Therefore, a massive vertex like (2.28) cannot possibly be in the cohomology of the BRST charge (2.23). However, as we will present in the next section, there is an alternative formulation of the heterotic sectorized string that indeed contains a massive solution.

\section{Alternative formulation}

By examining the structure of the BRST transformation (2.29), we can directly identify the origin of the wave operator, $\square$. It comes from the piece $\bar{c} T_{+}$of the BRST charge. Had 
it been replaced by $\bar{c} T_{-}$, the wave operator would change sign, potentially leading to a massive state in the cohomology. At this point, we observe an ambiguity in defining the $(+)$ and $(-)$ sectors of the heterotic model. Notice that instead of the operator set $(2.21)$, we can define

$$
\begin{aligned}
& \hat{d}_{\alpha}=p_{\alpha}-\frac{1}{2} P_{m}\left(\gamma^{m} \theta\right)_{\alpha}-\frac{1}{2 \ell^{2}} \Pi^{m}\left(\gamma_{m} \theta\right)_{\alpha}, \\
& \hat{P}_{m}^{+}=P_{m}+\frac{1}{\ell^{2}} \partial X_{m}+\frac{1}{\ell^{2}}\left(\theta \gamma_{m} \partial \theta\right), \\
& \hat{P}_{m}^{-}=P_{m}-\frac{1}{\ell^{2}} \partial X_{m},
\end{aligned}
$$

and, as the characteristic energy-momentum tensors of each sector,

$$
\begin{aligned}
& \hat{T}_{+} \equiv-\frac{1}{4} \eta^{m n} \hat{P}_{m}^{+} \hat{P}_{n}^{+}-\frac{1}{\ell^{2}}\left(\hat{d}_{\alpha} \partial \theta^{\alpha}+w_{\alpha} \partial \lambda^{\alpha}\right), \\
& \hat{T}_{-} \equiv \frac{1}{4} \eta^{m n} \hat{P}_{m}^{-} \hat{P}_{n}^{-}-\frac{1}{\ell^{2}}\left(2 \bar{b} \partial \bar{c}-\bar{c} \partial \bar{b}-T_{C}\right) .
\end{aligned}
$$

As before, the total energy-momentum tensor can be expressed as $T=\ell^{2}\left(\hat{T}_{+}+\hat{T}_{-}\right)$.

Therefore, we are naturally led to two different models (A and B, from now on) depending on the realization of the supersymmetry algebra, $\left\{q_{\alpha}, q_{\beta}\right\}=-\gamma_{\alpha \beta}^{m} \partial_{m}$, with

$$
\begin{aligned}
& q_{\alpha}^{A}=\oint\left\{p_{\alpha}+\frac{1}{2} P_{m}\left(\gamma^{m} \theta\right)_{\alpha}-\frac{1}{2 \ell^{2}} \partial X^{m}\left(\gamma_{m} \theta\right)_{\alpha}-\frac{1}{12 \ell^{2}}\left(\theta \gamma^{m} \partial \theta\right)\left(\gamma_{m} \theta\right)_{\alpha}\right\}, \\
& q_{\alpha}^{B}=\oint\left\{p_{\alpha}+\frac{1}{2} P_{m}\left(\gamma^{m} \theta\right)_{\alpha}+\frac{1}{2 \ell^{2}} \partial X^{m}\left(\gamma_{m} \theta\right)_{\alpha}+\frac{1}{12 \ell^{2}}\left(\theta \gamma^{m} \partial \theta\right)\left(\gamma_{m} \theta\right)_{\alpha}\right\},
\end{aligned}
$$

and the heterotic sectorized model is two-fold, resembling the two possible descriptions of the heterotic chiral string studied in [12]. ${ }^{4}$ The A model was discussed in section 2 and its spectrum contains only massless states. The B model will be defined by the BRST charge

$$
\begin{aligned}
\hat{Q} & =\hat{Q}_{\lambda}+Q_{-}, \\
\hat{Q}_{\lambda} & \equiv \oint \lambda^{\alpha} \hat{d}_{\alpha}, \\
Q_{-} & \equiv \oint \bar{c}\left(\hat{T}_{-}+\frac{1}{\ell^{2}} \bar{b} \partial \bar{c}\right),
\end{aligned}
$$

cf. equations (3.1) and (3.2). Nilpotency of $\hat{Q}$ is straightforward to demonstrate and its physical content will be discussed next.

\subsection{Cohomology}

The massless cohomology in the B model is easily obtained, having the same physical content of the A model. The ghost number two vertex operators are given by

$$
\begin{aligned}
U_{\mathrm{SYM}} & =\lambda^{\alpha} \bar{c} A_{\alpha}^{I} J_{I}, \\
U_{\mathrm{SG}} & =\lambda^{\alpha} \bar{c} A_{\alpha}^{m} \hat{P}_{m}^{-},
\end{aligned}
$$

\footnotetext{
${ }^{4}$ Note that the only other possible realization of the supersymmetry generator is $q_{\alpha}=\oint\left\{p_{\alpha}+\right.$ $\left.\frac{1}{2} P_{m}\left(\gamma^{m} \theta\right)_{\alpha}\right\}$, which corresponds to the ambitwistor case and to which $q_{\alpha}^{A}$ and $q_{\alpha}^{B}$ converge in the $\ell \rightarrow \infty$ limit.
} 
with equations of motion and gauge transformations analogous to the ones displayed in (2.8) and (2.9).

Now we will consider a vertex operator of the form $U=\bar{c} U_{\text {open }}$, with

$$
\begin{aligned}
U_{\text {open }} \equiv & \lambda^{\alpha} \partial \theta^{\beta} B_{\alpha \beta}+\left(\hat{d}_{\beta}, \lambda^{\alpha} C_{\alpha}^{\beta}\right)+\left(J, \lambda^{\alpha} E_{\alpha}\right) \\
& +\left(N^{m n}, \lambda^{\alpha} F_{\alpha m n}\right)+\partial \lambda^{\alpha} G_{\alpha}+\left(\hat{P}_{m}^{+}, \lambda^{\alpha} H_{\alpha}^{m}\right) .
\end{aligned}
$$

Here, $J=-w_{\alpha} \lambda^{\alpha}$ is the ghost number current associated to the pure spinor conjugate pair and $N^{m n}=-\frac{1}{2}\left(w \gamma^{m n} \lambda\right)$ is the ghost Lorentz contribution. The superfields $B_{\alpha \beta}, C_{\alpha}^{\beta}, E_{\alpha}$, $F_{\alpha m n}, G_{\alpha}$ and $H_{\alpha}^{m}$ were conveniently chosen to resemble the covariant description of the first massive level of the pure spinor open string in [18]. Due to the pure spinor constraint, not all superfields in $U_{\text {open }}$ are independent, for it implies that

$$
\left(N^{m n}, \lambda^{\alpha}\right) \gamma_{\alpha \beta}^{p} \eta_{n p}+\frac{1}{2}\left(J, \lambda^{\alpha}\right) \gamma_{\alpha \beta}^{m}=-2 \gamma_{\alpha \beta}^{m} \partial \lambda^{\alpha} .
$$

Therefore, $U_{\text {open }}$ is invariant under the transformations parametrized by an arbitrary superfield $\Omega_{m}^{\alpha}$,

$$
\begin{aligned}
\delta E_{\alpha} & =\gamma_{\alpha \beta}^{m} \Omega_{m}^{\beta}, \\
\delta F_{\alpha m n} & =\eta_{n p} \gamma_{\alpha \beta}^{p} \Omega_{m}^{\beta}-\eta_{m p} \gamma_{\alpha \beta}^{p} \Omega_{n}^{\beta}, \\
\delta G_{\alpha} & =4 \gamma_{\alpha \beta}^{m} \Omega_{m}^{\beta} .
\end{aligned}
$$

Now we can turn to the action of the BRST charge on $U$,

$$
[\hat{Q}, U]=-\bar{c}\left\{\hat{Q}_{\lambda}, U_{\text {open }}\right\}+\left(\frac{1}{\ell^{2}}-\frac{\square}{4}\right) \bar{c} \partial \bar{c} U_{\text {open }} .
$$

As before, BRST-closedness requires that the two terms on the right hand side above vanish independently. Being careful with the ordering prescription, one can show that

$$
\begin{aligned}
\left\{\hat{Q}_{\lambda}, U_{\text {open }}\right\}= & \lambda^{\alpha} \lambda^{\gamma} \partial \theta^{\beta}\left(-D_{\gamma} B_{\alpha \beta}+\frac{2}{\ell^{2}} \gamma_{\beta \gamma}^{m} H_{\alpha}^{n} \eta_{m n}\right) \\
& +\left(\hat{P}_{m}^{+}, \lambda^{\alpha} \lambda^{\beta}\left[D_{\beta} H_{\alpha}^{m}-\gamma_{\beta \gamma}^{m} C_{\alpha}^{\gamma}\right]\right) \\
& -\left(\hat{d}_{\beta}, \lambda^{\alpha} \lambda^{\gamma}\left[\delta_{\gamma}^{\beta} E_{\alpha}+D_{\gamma} C_{\alpha}^{\beta}+\frac{1}{2}\left(\gamma^{m n}\right)_{\gamma}^{\beta} F_{\alpha m n}\right]\right) \\
& +\left(J, \lambda^{\alpha} \lambda^{\beta} D_{\beta} E_{\alpha}\right)+\left(N^{m n}, \lambda^{\alpha} \lambda^{\beta} D_{\beta} F_{\alpha m n}\right) \\
& +\lambda^{\alpha} \partial \lambda^{\beta}\left(B_{\alpha \beta}+D_{\alpha} G_{\beta}+\gamma_{\beta \gamma}^{m} \partial_{m} C_{\alpha}^{\gamma}\right) \\
& -\lambda^{\alpha} \partial \lambda^{\beta}\left(D_{\beta} E_{\alpha}+\frac{1}{2}\left(\gamma^{m n}\right)_{\beta}^{\gamma} D_{\gamma} F_{\alpha m n}\right)
\end{aligned}
$$

and the vanishing of the above expression immediately implies the superfield equations of motion

$$
\begin{aligned}
\lambda^{\alpha} \lambda^{\beta}\left(D_{\beta} H_{\alpha}^{m}-\gamma_{\beta \gamma}^{m} C_{\alpha}^{\gamma}\right) & =0, \\
\lambda^{\alpha} \lambda^{\gamma}\left(D_{\gamma} B_{\alpha \beta}-\frac{2}{\ell^{2}} \gamma_{\beta \gamma}^{m} H_{\alpha}^{n} \eta_{m n}\right) & =0, \\
\lambda^{\alpha} \lambda^{\gamma}\left(\delta_{\gamma}^{\beta} E_{\alpha}+D_{\gamma} C_{\alpha}^{\beta}+\frac{1}{2}\left(\gamma^{m n}\right)_{\gamma}^{\beta} F_{\alpha m n}\right) & =0 .
\end{aligned}
$$


Again, not all the terms in (3.11) are independent as can be seen from the identity:

$$
\left(N^{m n}, \lambda^{\alpha} \lambda^{\beta}\right) \gamma_{\gamma \beta}^{p} \eta_{n p}+\frac{1}{2}\left(J, \lambda^{\alpha} \lambda^{\beta}\right) \gamma_{\gamma \beta}^{m}=-\frac{5}{2}\left(\gamma^{m} \partial \lambda\right)_{\gamma} \lambda^{\alpha}-\frac{1}{2}\left(\gamma^{m n} \lambda\right)^{\alpha}\left(\gamma_{n} \partial \lambda\right)_{\gamma}
$$

Taking this into account, the vanishing of $\left\{\hat{Q}_{\lambda}, U_{\text {open }}\right\}$ also implies

$$
\begin{aligned}
& \lambda^{\alpha} \lambda^{\beta} D_{\beta} E_{\alpha}=\left(\lambda \gamma_{m p q r s} \lambda\right) K_{n}^{p q r s} \eta^{m n}, \\
& \lambda^{\alpha} \lambda^{\beta} D_{\beta} F_{\alpha m n}=\frac{1}{2}\left(\lambda \gamma_{n p q r s} \lambda\right) K_{m}^{p q r s}-\frac{1}{2}\left(\lambda \gamma_{m p q r s} \lambda\right) K_{n}^{p q r s}, \\
& \lambda^{\alpha} \partial \lambda^{\beta}\left(B_{\alpha \beta}+D_{\alpha} G_{\beta}-D_{\beta} E_{\alpha}-\frac{1}{2}\left(\gamma^{m n}\right)_{\beta}^{\gamma} D_{\gamma} F_{\alpha m n}+\gamma_{\beta \gamma}^{m} \partial_{m} C_{\alpha}^{\gamma}\right) \\
&=-2 \lambda^{\alpha} \partial \lambda^{\beta}\left(\gamma^{m} \gamma_{n p q r}\right)_{\beta \alpha} K_{m}^{n p q r}+8 \lambda^{\alpha} \partial \lambda^{\beta}\left(\gamma_{n p q}\right)_{\beta \alpha} K_{m}^{n p q m},
\end{aligned}
$$

where $K_{m}^{n p q r}$ is an arbitrary superfield.

Concerning BRST-exact contributions, $U_{\text {open }}$ is defined up to the following transformations

$$
\begin{aligned}
\delta B_{\alpha \beta} & =D_{\alpha} \Sigma_{\beta}+\frac{2}{\ell^{2}} \gamma_{\alpha \beta}^{n} \Sigma^{m} \eta_{m n}, \\
\delta C_{\alpha}^{\beta} & =\delta_{\alpha}^{\beta} \Sigma+\frac{1}{2}\left(\gamma^{m n}\right)^{\beta}{ }_{\alpha} \Sigma_{m n}+D_{\alpha} \Sigma^{\beta}, \\
\delta E_{\alpha} & =-D_{\alpha} \Sigma, \\
\delta F_{\alpha m n} & =-D_{\alpha} \Sigma_{m n}, \\
\delta G_{\alpha} & =D_{\alpha} \Sigma-\Sigma_{\alpha}-\gamma_{\alpha \beta}^{m} \partial_{m} \Sigma^{\beta}+\frac{1}{2}\left(\gamma^{m n}\right)_{\alpha}^{\beta} D_{\beta} \Sigma_{m n}, \\
\delta H_{\alpha}^{m} & =\gamma_{\alpha \beta}^{m} \Sigma^{\beta}+D_{\alpha} \Sigma^{m} .
\end{aligned}
$$

Here, $\Sigma, \Sigma_{\alpha}, \Sigma^{\alpha}, \Sigma^{m}$ and $\Sigma_{m n}$ are superfield parameters. It is not difficult to show that the superfield equations of motion in (3.12) and (3.14) are invariant under (3.15).

Up to irrelevant constants, the very same equations of motion were obtained in [18] in a different context, corresponding to a superfield description of the first massive level of the open string. In our case, the mass is given in terms of the dimensionful parameter $\ell$, such that $M^{2}=\frac{4}{\ell^{2}}$. This will be proven below, precisely matching the remaining condition for the vertex $U$ to be BRST-closed, cf. equation (3.10). Note that it is not possible to accommodate any other massive states in the cohomology of the heterotic B model and such massive level only exists because of a simple interplay between the two sectors.

The presence of an open string like state, i.e. a state matching the quantum numbers of the first massive level of the open string, is characteristic to the sectorized models. Next, we will further explore this feature, discussing the field content of the massive cohomology and determining the physical degrees of freedom in order to explicitly write their vertex operator in the light-cone gauge. 


\subsection{Light-cone description of the massive states}

The first massive level of the open string consists of a symmetric, traceless tensor, $G_{m n}$, a 3 -form, $A_{m n p}$ and a $\gamma$-traceless vector-spinor, $\psi_{m \alpha}$, satisfying

$$
\begin{aligned}
\eta^{m n} G_{m n} & =0, \\
\partial^{m} G_{m n} & =0, \\
\partial^{m} \psi_{m \alpha} & =0, \\
\left(\gamma^{m} \psi_{m}\right)^{\alpha} & =0, \\
\partial^{m} A_{m n p} & =0 .
\end{aligned}
$$

These equations are invariant under the $\mathcal{N}=1$ supersymmetry transformations

$$
\begin{aligned}
\delta G_{m n}= & M\left(\epsilon \gamma_{m} \chi_{n}\right)+M\left(\epsilon \gamma_{n} \chi_{m}\right)-\left(\epsilon \partial_{m} \psi_{n}\right)-\left(\epsilon \partial_{n} \psi_{m}\right) \\
\delta \psi_{m \alpha}= & \frac{1}{4} G_{m n}\left(\gamma^{n} \epsilon\right)_{\alpha}+\frac{1}{4} \partial_{q} A_{m n p}\left(\gamma^{n p q} \epsilon\right)_{\alpha}-\frac{1}{24} \partial_{r} A_{n p q}\left(\gamma_{m}{ }^{n p q r} \epsilon\right)_{\alpha} \\
\delta A_{m n p}= & \frac{1}{3}\left[\left(\epsilon \gamma_{m n} \psi_{p}\right)+\left(\epsilon \gamma_{n p} \psi_{m}\right)+\left(\epsilon \gamma_{p m} \psi_{n}\right)\right] \\
& -\frac{1}{3 M}\left[\left(\epsilon \gamma_{m} \partial_{n} \chi_{p}\right)+\left(\epsilon \gamma_{n} \partial_{p} \chi_{m}\right)+\left(\epsilon \gamma_{p} \partial_{m} \chi_{n}\right)\right] \\
& +\frac{1}{3 M}\left[\left(\epsilon \gamma_{n} \partial_{m} \chi_{p}\right)+\left(\epsilon \gamma_{p} \partial_{n} \chi_{m}\right)+\left(\epsilon \gamma_{m} \partial_{p} \chi_{n}\right)\right],
\end{aligned}
$$

where $\epsilon^{\alpha}$ is a constant fermionic parameter, $M$ is the mass and $\chi_{m}^{\alpha} \equiv \frac{1}{M} \gamma_{n}^{\alpha \beta} \partial^{n} \psi_{m \beta}$.

The supersymmetry algebra closes on-shell,

$$
\begin{aligned}
{\left[\delta_{1}, \delta_{2}\right] G_{m n} } & =\left(\epsilon_{1} \gamma^{p} \epsilon_{2}\right) \partial_{p} G_{m n}, \\
{\left[\delta_{1}, \delta_{2}\right] \psi_{m \alpha} } & =\left(\epsilon_{1} \gamma^{n} \epsilon_{2}\right) \partial_{n} \psi_{m \alpha}, \\
{\left[\delta_{1}, \delta_{2}\right] A_{m n p} } & =\left(\epsilon_{1} \gamma^{q} \epsilon_{2}\right) \partial_{q} A_{m n p},
\end{aligned}
$$

and the matching of the on-shell degrees of freedom (d.o.f.) can be readily verified. There are 128 bosonic d.o.f. ( 44 from $G^{m n}$ and 84 from $A_{m n p}$ ) and 128 fermionic d.o.f. coming from $\psi_{m \alpha}$. It is possible of course to extract all this information about the physical spectrum from the covariant superfield formulation [18], but the demonstration is lengthy and purposeless for our present goal.

Alternatively, we will take advantage of the DDF-like construction of [19] and propose an $\mathrm{SO}(8)$ covariant version of $U_{\text {open }}$ given in (3.7). This will allow us to pinpoint the physical polarizations and to have a better knowledge of their behaviour in the $\ell \rightarrow \infty$ limit that will be analyzed in the next section. In order to do it properly, we will need a quick review of the $\mathrm{SO}(8)$ notation. Spacetime vectors, $v^{m}$, will be decomposed in transversal components, $v^{i}$, with $i=1, \ldots, 8$, and light-cone components, $\sqrt{2} v^{ \pm}=\left(v^{0} \pm v^{9}\right)$. Spacetime spinors, $s^{\alpha}\left(t_{\alpha}\right)$, will be written in terms of the two $\mathrm{SO}(8)$ chiralities, $s^{a}$ and $\bar{s}^{\dot{a}}\left(t_{a}\right.$ and $\left.\bar{t}_{\dot{a}}\right)$, with $a, \dot{a}=1, \ldots, 8$. To keep the notation simple, we will use only diagonal $\mathrm{SO}(8)$ metrics, $\left\{\eta_{i j}, \eta_{a b}, \eta_{\dot{a} \dot{b}}\right\}$, and their inverse, $\left\{\eta^{i j}, \eta^{a b}, \eta^{\dot{a} \dot{b}}\right\}$, making no distinction between upper and 
lower indices. The matrices $\gamma^{m}$ will be conveniently written in terms of the 8-dimensional equivalent of the Pauli matrices, $\sigma_{a \dot{a}}^{i}$, with non-vanishing components given by

$$
\begin{array}{llrl}
\gamma_{\alpha \beta}^{i} & =\sigma_{a \dot{a}}^{i}, & \left(\gamma^{i}\right)^{\alpha \beta}=\sigma_{b \dot{b}}^{i} \eta^{a b} \eta^{\dot{a} \dot{b}}, \\
\gamma_{\alpha \beta}^{+}=-\sqrt{2} \eta_{a b}, & \left(\gamma^{+}\right)^{\alpha \beta}=\sqrt{2} \eta^{\dot{a} \dot{b}}, \\
\gamma_{\alpha \beta}^{-}=-\sqrt{2} \eta_{\dot{a} \dot{b}}, & \left(\gamma^{-}\right)^{\alpha \beta}=\sqrt{2} \eta^{a b},
\end{array}
$$

Note that the $\sigma^{i}$ matrices satisfy

$$
\begin{aligned}
\left(\sigma_{a \dot{a}}^{i} \sigma_{b \dot{b}}^{j}+\sigma_{a \dot{b}}^{i} \sigma_{b \dot{a}}^{j}\right) \eta_{i j} & =2 \eta_{a b} \eta_{\dot{a} \dot{b}}, \\
\left(\sigma_{a \dot{a}}^{i} \sigma_{b \dot{b}}^{j}+\sigma_{a \dot{b}}^{i} \sigma_{b \dot{a}}^{j}\right) \eta^{\dot{a} \dot{b}} & =2 \eta^{i j} \eta_{a b}, \\
\left(\sigma_{a \dot{a}}^{i} \sigma_{b \dot{b}}^{j}+\sigma_{a \dot{b}}^{i} \sigma_{b \dot{a}}^{j}\right) \eta^{a b} & =2 \eta^{i j} \eta_{\dot{a} \dot{b}} .
\end{aligned}
$$

It will be useful to define also $\sigma^{i j} \equiv \frac{1}{2}\left(\sigma^{i} \sigma^{j}-\sigma^{j} \sigma^{i}\right)$, which can be viewed as the spinor Lorentz matrices in $\mathrm{SO}(8)$.

Next, we define the $\mathrm{SO}(8)$-covariant superfield

$$
F_{\dot{a} a}(a, \bar{a}, \xi, \bar{\xi}, k, p) \equiv \bar{A}_{\dot{a}}(\bar{a}, \bar{\xi}, k) A_{a}(a, \xi, p),
$$

with

$$
\begin{aligned}
\bar{A}_{\dot{a}}(\bar{a}, \bar{\xi}, k)= & \left\{\eta_{i l}-\frac{i k}{3 !} \theta_{i l}-\frac{k^{2}}{5 !} \theta_{i j} \theta_{j l}+\frac{i k^{3}}{7 !} \theta_{i j} \theta_{j k} \theta_{k l}\right\} \bar{a}^{i}\left(\sigma^{l} \theta\right)_{\dot{a}} e^{-i k \sqrt{2} X^{+}}+\frac{i}{k} \bar{\xi}_{\dot{a}} e^{-i k \sqrt{2} X^{+}} \\
& +\left\{\frac{1}{2 !} \eta_{i l}-\frac{i k}{4 !} \theta_{i l}-\frac{k^{2}}{6 !} \theta_{i j} \theta_{j l}+\frac{i k^{3}}{8 !} \theta_{i j} \theta_{j k} \theta_{k l}\right\}\left(\bar{\xi} \sigma^{i} \theta\right)\left(\sigma^{l} \theta\right)_{\dot{a}} e^{-i k \sqrt{2} X^{+}} \\
A_{a}(a, \xi, p)= & \left\{\eta_{i l}-\frac{i p}{3 !} \bar{\theta}_{i l}-\frac{p^{2}}{5 !} \bar{\theta}_{i j} \bar{\theta}_{j l}+\frac{i p^{3}}{7 !} \bar{\theta}_{i j} \bar{\theta}_{j k} \bar{\theta}_{k l}\right\} a^{i}\left(\sigma^{l} \bar{\theta}\right)_{a} e^{-i p \sqrt{2} X^{-}}+\frac{i}{p} \xi_{a} e^{-i p \sqrt{2} X^{-}} \\
& +\left\{\frac{1}{2 !} \eta_{i l}-\frac{i p}{4 !} \bar{\theta}_{i l}-\frac{p^{2}}{6 !} \bar{\theta}_{i j} \bar{\theta}_{j l}+\frac{i p^{3}}{8 !} \bar{\theta}_{i j} \bar{\theta}_{j k} \bar{\theta}_{k l}\right\}\left(\xi \sigma^{i} \bar{\theta}\right)\left(\sigma^{l} \bar{\theta}\right)_{a} e^{-i p \sqrt{2} X^{-}}
\end{aligned}
$$

These objects correspond to the $\mathrm{SO}(8)$-covariant super Maxwell fields of [20], with $\left(\bar{a}_{i}, \bar{\xi}_{\dot{a}}\right)$ and $\left(a_{i}, \xi_{a}\right)$ denoting the polarization pairs for the vector and the spinor in each frame. Note that $\bar{A}_{\dot{a}}\left(A_{a}\right)$ depends on $\theta_{a}\left(\bar{\theta}_{\dot{a}}\right)$ only through $\theta^{i j}=\theta_{a} \sigma_{a b}^{i j} \theta_{b}\left(\bar{\theta}^{i j}=\bar{\theta}_{\dot{a}} \sigma_{\dot{a} \dot{b}}^{i j} \bar{\theta}_{\dot{b}}\right)$. The vector superfields are defined by

$$
\begin{aligned}
& D_{a} \bar{A}_{\dot{a}}(k)=\sigma_{a \dot{a}}^{i} \bar{A}_{i}(k), \\
& \bar{D}_{\dot{a}} A_{a}(p)=\sigma_{a \dot{a}}^{i} A_{i}(p),
\end{aligned}
$$

and can be used to show that $F_{a \dot{a}}$ defines a superfield set $\left\{F_{a \dot{a}}, F_{i a}, F_{\dot{a} i}, F_{i j}\right\}$ satisfying

$$
\begin{aligned}
D_{b} F_{\dot{a} a} & =\sigma_{b \dot{a}}^{i} F_{i a}, & & D_{a} F_{\dot{a} i}=\sigma_{a \dot{a}}^{j} F_{j i}, \\
\bar{D}_{\dot{b}} F_{\dot{a} a} & =-\sigma_{\dot{b} a}^{i} F_{\dot{a} i}, & & \bar{D}_{\dot{b}} F_{\dot{a} i}=-i p \sigma_{\dot{b} a}^{i} F_{\dot{a} a}, \\
D_{b} F_{i a} & =i k \sigma_{b \dot{a}}^{i} F_{\dot{a} a}, & & D_{a} F_{i j}=i k \sigma_{a \dot{a}}^{i} F_{\dot{a} j}, \\
\bar{D}_{\dot{a}} F_{i a} & =\sigma_{a \dot{a}}^{j} F_{i j}, & & \bar{D}_{\dot{a}} F_{i j}=i p \sigma_{a \dot{a}}^{j} F_{i a},
\end{aligned}
$$

which will be the fundamental blocks in the construction of the $\mathrm{SO}(8)$-covariant form of $U_{\text {open }}$. To build it explicitly, we can just go through the DDF-like procedure described 
in [19] for generating the massive vertices and adapt it to the sectorized model. The result is

$$
\begin{aligned}
U_{\text {open }}(a, \bar{a}, \xi, \bar{\xi}, k, p)= & \left(\hat{P}_{i}^{+}, \bar{\lambda}_{\dot{a}} F_{\dot{a} i}\right)-\frac{1}{\sqrt{2}} \sigma_{a \dot{a}}^{i}\left(\hat{P}_{+}^{+}, \lambda_{a} F_{\dot{a} i}\right)-i p \sqrt{2}\left(N_{i}, \bar{\lambda}_{\dot{a}} F_{\dot{a} i}\right) \\
& -\frac{2}{\ell^{2}} \bar{\lambda}_{\dot{a}} \partial \theta_{a} F_{\dot{a} a}+i p\left(\bar{\lambda} \sigma^{j} \sigma^{i} \partial \bar{\theta}\right) F_{i j}-i p\left(\hat{d}_{a}, \bar{\lambda}_{\dot{a}} F_{\dot{a} a}\right) .
\end{aligned}
$$

Here, $\hat{P}_{i}^{+}$and $\hat{P}_{+}^{+}$are just components of $\hat{P}_{m}^{+}$and $N^{i} \equiv N^{-i}$. The above expression corresponds to the vertex depicted in (3.7) in the light-cone frame, after the removal of auxiliary superfields through (3.9) and (3.15). It is now simple to determine the mass shell, since

$$
\begin{aligned}
\left\{\hat{Q}_{\lambda}, U_{\text {open }}\right\}= & 2\left(k p-\frac{1}{\ell^{2}}\right)(\bar{\lambda} \partial \bar{\theta}) \lambda^{a} \sigma_{a \dot{a}}^{i} F_{\dot{a} i}-2\left(k p-\frac{1}{\ell^{2}}\right)\left(\lambda \sigma^{i} \partial \bar{\theta}\right) \bar{\lambda}_{\dot{a}} F_{\dot{a} i} \\
& +2\left(k p-\frac{1}{\ell^{2}}\right) \bar{\lambda}_{\dot{a}} \partial \lambda_{a} F_{\dot{a} a}
\end{aligned}
$$

which vanishes only for $k p=\frac{1}{\ell^{2}}$, i.e. $M^{2}=\frac{4}{\ell^{2}}$. Therefore, the vertex $U=\bar{c} U_{\text {open }}$ describes physical states in the massive cohomology of the B model.

The physical degrees of freedom are easily readable from (3.26). Let us extract the vertex operators from each polarization set by defining

$$
\begin{aligned}
U_{j, i} & \equiv \frac{\partial}{\partial \bar{a}_{i}} \frac{\partial}{\partial a^{j}} U, & U_{a, i} & \equiv \frac{\partial}{\partial \bar{a}_{i}} \frac{\partial}{\partial \xi^{a}} U \\
U_{j, \dot{a}} & \equiv \frac{\partial}{\partial \bar{\xi}_{\dot{a}}} \frac{\partial}{\partial a^{j}} U, & U_{a, \dot{a}} & \equiv \frac{\partial}{\partial \bar{\xi}_{\dot{a}}} \frac{\partial}{\partial \xi^{a}} U .
\end{aligned}
$$

The symmetric part of $U_{j, i}$ and the vector $\sigma_{a \dot{a}}^{i} U_{a, \dot{a}}$ combine to give an $\mathrm{SO}(9)$ symmetric traceless tensor of rank 2, with 44 d.o.f. The antisymmetric part of $U_{j, i}$ and the 3form $\sigma_{a \dot{a}}^{i j k} U_{a, \dot{a}}$ combine into an $\mathrm{SO}(9) 3$-form $\left(\sigma^{i j k}\right.$ is just the antisymmetrized product of three $\sigma$ matrices), with 84 d.o.f. . As for the 128 fermionic degrees of freedom, $U_{a, i}$ and $U_{j, \dot{a}}$ constitute a $\gamma$-traceless $\mathrm{SO}(9)$ vector-spinor. These are the irreducible massive representations of the fields $G_{m n}, A_{m n p}$ and $\psi_{m \alpha}$, respectively, with equations of motion given in 3.16. This finishes the demonstration that the heterotic B model in the sectorized string contains a massive open string like state.

In the next section we will analyze how the sectorized model is connected to the ambitwistor string, showing what happens to the massive degrees of freedom in the infinite length limit.

\section{The $\ell \rightarrow \infty$ limit and the connection to ambitwistor strings}

In the ambitwistor string debutant paper [2], Mason and Skinner pointed out that the model remarkably shares features of both $\alpha^{\prime} \rightarrow 0$ (infinite tension string) and $\alpha^{\prime} \rightarrow \infty$ (null string) limits. This was further explored in [11] to determine a connection between the ambitwistor string and the null string. In this section we will give an explicit construction of 
the $\ell \rightarrow \infty$ limit, showing that the ambitwistor string can be viewed as a zero tension limit of the sectorized model. Although we are focusing on the heterotic case, this construction is easily generalized to the type II string and the bosonic string.

\subsection{The heterotic ambitwistor string in the pure spinor formalism}

By taking the limit $\ell \rightarrow \infty$ in the B model described in the previous section, it is easy to see that the BRST charge (3.4a) reduces to

$$
Q=\oint\left\{\lambda^{\alpha} d_{\alpha}+\frac{1}{4} \bar{c} P^{m} P_{m}\right\}
$$

now with $d_{\alpha}=p_{\alpha}-\frac{1}{2}\left(\gamma^{m} \theta\right)_{\alpha} P_{m}$. In the $\mathrm{A}$ model, the only difference is a minus sign in front of the $P^{2}$ term. $Q$ should be regarded as the BRST charge for the heterotic ambitwistor string in the pure spinor formalism, in which the constraint associated to the $(\bar{b}, \bar{c})$ ghost pair is the particle-like Hamiltonian, $H=-\frac{1}{2} P^{2}$. For comparison, in Berkovits' proposal [3], $\bar{b}$ and $\bar{c}$ are identified with the (left-moving) Virasoro ghosts. While both are consistent at the quantum level, the latter does not completely describe the supergravity states by failing to express the expected gauge transformations as BRST-exact states.

It is interesting to mention that the difference between (4.1) and the BRST charges of the A and B models is the nilpotent symmetry of the action (2.1) defined in [7] (which was an extension of the type II construction of $[5,6])$, generated by

$$
\mathcal{K}=\frac{1}{\ell^{2}} \oint\left\{\left(\lambda \gamma_{m} \theta\right) \Pi^{m}\right\}
$$

Because of the dimensionful parameter $\ell, \mathcal{K}$ cannot be part of the BRST charge in the infinite length limit. ${ }^{5}$

Note also that $T=\ell^{2}\left(\hat{T}_{+}+\hat{T}_{-}\right)$and $\mathcal{H}=\left(\hat{T}_{+}-\hat{T}_{-}\right)$satisfy

$$
\begin{aligned}
T(z) T(y) & \sim \frac{2 T}{(z-y)^{2}}+\frac{\partial T}{(z-y)}, \\
T(z) \mathcal{H}(y) & \sim \frac{2 \mathcal{H}}{(z-y)^{2}}+\frac{\partial \mathcal{H}}{(z-y)}, \\
\mathcal{H}(z) \mathcal{H}(y) & \sim \frac{1}{\ell^{2}} \frac{2 T}{(z-y)^{2}}+\frac{1}{\ell^{2}} \frac{\partial T}{(z-y)},
\end{aligned}
$$

which in the $\ell \rightarrow \infty$ limit corresponds to the Galilean Conformal Algebra [21] and appears as the constraint algebra in the tensionless string [22]. The OPE's in (4.3) are characteristic to the sectorized strings [7] and in fact were later briefly considered as part of a tensionful model whose tensionless limit gives the ambitwistor bosonic string [11].

Concerning the tree-level amplitudes discussed in section 2, the introduction of the dimensionful parameter $\ell$ adds a factor of $\ell^{-2}$ to the $P^{+} P^{+}$OPE, which ultimately implies

\footnotetext{
${ }^{5}$ This might help to understand how to consistently couple the supergravity background to the ambitwistor string in the pure spinor formulation, task that so far has only been achieved in the sectorized model.
} 
that the Einstein-gravity term $\mathcal{M}_{1}$ appears multiplied by the same factor. The calculations in the A and B models are essentially the same and can be schematically cast as

$$
\left\langle U_{1} U_{2} U_{3}\right\rangle= \pm \frac{1}{\ell^{2}}(\text { Einstein })+(\text { Gauss-Bonnet }),
$$

where the plus sign corresponds to the A model and the minus sign corresponds to the $\mathrm{B}$ model. This is consistent with the results of [12], where the ambiguity comes from the different choices of "flipped" sector, and is also consistent with the interpretation of the ambitwistor string as the $\ell \rightarrow \infty$ limit of the sectorized string, since the graviton threepoint function in the heterotic ambitwistor model gives precisely a Gauss-Bonnet term at tree level.

The cohomology of (4.1) should contain a manifestly supersymmetric version of the spectrum found in [2]. We have of course the super Yang-Mills vertex, $U_{\mathrm{SYM}}=\lambda^{\alpha} \bar{c} A_{\alpha}^{I} J_{I}$, and supergravity vertex, $U_{\mathrm{SG}}=\lambda^{\alpha} \bar{c} A_{\alpha}^{m} P_{m}$, with the expected equations of motion and gauge transformations. In addition, Mason and Skinner showed that the heterotic ambitwistor cohomology contains also a 3-form so far of unknown origin. But there are possibly even more states previously unaccounted for. Maybe the easiest way to see this is determining the zero-momentum cohomology of (4.1). In particular, we can focus on the following vertices,

$$
\begin{aligned}
U_{m} \equiv & \bar{c}\left(\lambda \gamma^{n} \theta\right)\left(d \gamma_{m n} \theta\right)+\bar{c}\left(\lambda \gamma_{m} \theta\right) J-\frac{3}{4} \bar{c} P^{n}\left(\lambda \gamma^{p} \theta\right)\left(\theta \gamma_{m n p} \theta\right), \\
U_{m n p} \equiv & \bar{c}\left(\lambda \gamma_{[m} \theta\right)\left(d \gamma_{n p]} \theta\right)-2 \bar{c}\left(\lambda \gamma_{[m} \theta\right) N_{n p]} \\
& -\frac{1}{2} \bar{c} P_{[m}\left(\theta \gamma_{n p] q} \theta\right)\left(\lambda \gamma^{q} \theta\right)+\frac{1}{2} \bar{c} P^{q}\left(\lambda \gamma_{[m} \theta\right)\left(\theta \gamma_{n p] q} \theta\right),
\end{aligned}
$$

which are BRST-closed and cannot be written as BRST-exact operators.

As we will see next, such states can be interpreted as a massless limit of the first massive level of the open string, following the discussion in section 3 .

\subsection{The light-cone analysis of the ambitwistor cohomology}

If we consider a general vertex operator of the form

$$
U_{\infty}=\lim _{\ell \rightarrow \infty} \bar{c} U_{\text {open }},
$$

with $U_{\text {open }}$ given in (3.7), the equations of motion can be easily extracted from (3.12) and (3.14). So it seems that the supergravity vertex operator presented above is only part of a more general solution. To obtain such solution from scratch in its covariant form (including all the auxiliary superfields and associated gauge transformations) is again a cumbersome procedure. What we will do instead, is to solve the mass-shell constraint for the light-cone vertex (3.26) and then take the infinite length limit. Then, after identifying the relevant degrees of freedom, we will propose a covariantized version of the results.

Starting with (3.7) for $p=\frac{1}{k \ell^{2}}$ (BRST-closedness condition, cf. equation (3.27)), we can straightforwardly take the $\ell \rightarrow \infty$ limit and express the result as

$$
\lim _{\ell \rightarrow \infty} \bar{c} U_{\text {open }}(a, \bar{a}, \xi, \bar{\xi}, k)=a^{j} U_{j}(\bar{a}, \bar{\xi}, k)-\xi^{a} U_{a}(\bar{a}, \bar{\xi}, k),
$$


where

$$
\begin{aligned}
U_{j}(\bar{a}, \bar{\xi}, k) & =\left(P_{j}, \bar{c} \bar{\lambda}_{\dot{a}} \bar{A}_{\dot{a}}\right)-\frac{i}{k \sqrt{2}}\left\{Q,\left(P_{+}, \bar{c} \bar{A}_{j}\right)\right\} \\
U_{a}(\bar{a}, \bar{\xi}, k) & =\left(P_{i}, \bar{c} \bar{\lambda}_{\dot{a}} \bar{A}_{\dot{a}}\left(\sigma^{i} \bar{\theta}\right)_{a}\right)-\frac{1}{\sqrt{2}}\left(P_{+}, \bar{c}\left(\lambda \sigma^{i} \bar{A}\right)\left(\sigma^{i} \bar{\theta}\right)_{a}\right)+\left(d_{a}, \bar{c} \bar{\lambda}_{\dot{a}} \bar{A}_{\dot{a}}\right)+2 i k \bar{c} \partial \theta_{a} \bar{\lambda}_{\dot{a}} \bar{A}_{\dot{a}}
\end{aligned}
$$

At first glance, it is remarkable that $U_{\infty}$ can be so simply expressed in terms of the super Yang-Mills field $\bar{A}_{\dot{a}}$. This is a consequence of the DDF construction and does not seem to survive in the covariant formulation.

The vertex $U_{j}$, equation (4.7a), corresponds to a light-cone version of (3.6) and encodes the $\mathrm{SO}(8)$-covariant $\mathcal{N}=1$ heterotic supergravity multiplet up to a BRST exact term. By factoring out the polarizations, $U_{j}$ can be written as

$$
U_{j}(\bar{a}, \bar{\xi}, k)=\bar{a}_{i} U_{j, i}(k)+\bar{\xi}_{\dot{a}} U_{j, \dot{a}}(k) .
$$

The bosonic degrees of freedom (graviton, Kalb-Ramond field and dilaton) are described by $U_{j, i}$ while $U_{j, \dot{a}}$ is associated to their superpartners (gravitino and dilatino).

As a feature of the DDF construction, the vertex $U_{a}$ has the wrong Lorentz spin. This can be checked, for example, by analyzing the eigenvalue with respect to the Lorentz generator $\mathrm{M}^{+-}$of the lowest component in the vector polarization. A similar issue was discussed in [23] in the context of the DDF-like description of antifields in the pure spinor formalism. It can be corrected by rescaling one of the polarizations by the momentum factor $k$, such that the correct vertex should be defined in terms of $\bar{A}_{\dot{a}}\left(\frac{\bar{a}}{k}, \bar{\xi}, k\right)$ rather than $\bar{A}_{\dot{a}}(\bar{a}, \bar{\xi}, k)$. Finally, the vertex can be cast as

$$
U_{a}(\bar{a}, \bar{\xi}, k)=\bar{a}_{i} U_{a, i}(k)+\bar{\xi}_{\dot{a}} U_{a, \dot{a}}(k),
$$

having a simple supersymmetric structure,

$$
\begin{aligned}
{\left[q_{b}, U_{a, \dot{a}}\right] } & =k \sigma_{b \dot{a}}^{i} U_{a, i}, \\
\left\{q_{b}, U_{a, i}\right\} & =-i \sigma_{b \dot{a}}^{i} U_{a, \dot{a}} .
\end{aligned}
$$

The field content of $U_{a}$ can be readily determined and the $\mathrm{SO}(8)$ degrees of freedom are represented by a vector, $A_{i}$, a 3 -form, $A_{i j k}$, a spinor, $\bar{\psi}_{\dot{a}}$, and a sigma-traceless vectorspinor, $\psi_{a}^{i}$, with the following identification for the vertex operators:

$$
\begin{aligned}
A_{i} & \rightarrow \sigma_{a \dot{a}}^{i} U_{a, \dot{a}}, \\
A_{i j k} & \rightarrow \sigma_{a \dot{a}}^{i j k} U_{a, \dot{a}}, \\
\bar{\psi}_{\dot{a}} & \rightarrow \frac{1}{8} \sigma_{a \dot{a}}^{i} U_{a, i}, \\
\psi_{a}^{i} & \rightarrow U_{a, i}-\frac{1}{8} \sigma_{a \dot{a}}^{i}\left(\sigma_{\dot{a} b}^{j} U_{b, j}\right) .
\end{aligned}
$$

Note that the operators $U_{j}$ and $U_{a}$ are not completely unrelated, for they satisfy

$$
\begin{aligned}
{\left[\bar{q}_{\dot{a}}, U_{a}\right] } & =-\sigma_{a \dot{a}}^{j} U_{j}, \\
\left\{\bar{q}_{\dot{a}}, U_{j}\right\} & =0 .
\end{aligned}
$$



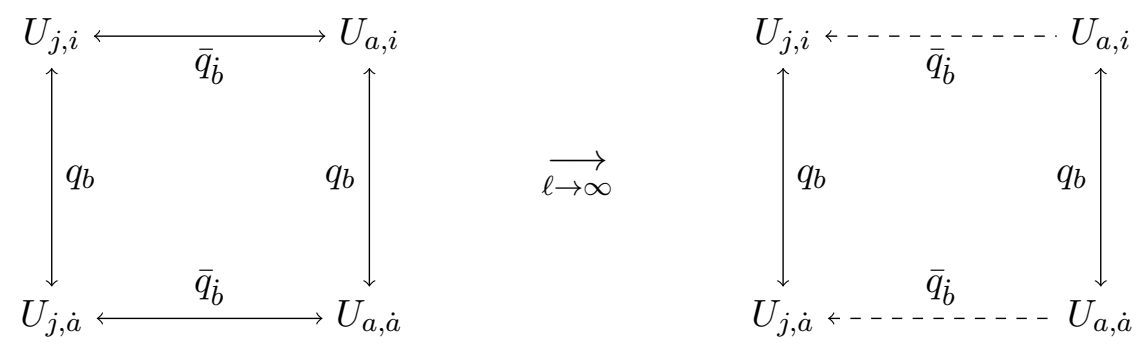

Figure 1. On the left we have the $\mathrm{SO}(8)$ degrees of freedom of the massive vertex (3.26). On the right side, after taking the limit $\ell \rightarrow \infty$, we are left with two partially independent massless multiplets, connected only by the algebra (4.12).

Therefore, knowing $U_{a}$ implies knowing $U_{j}$ although the opposite is not true. The above relations also convey an important information about the BRST triviality of $U_{a}$. Since the BRST charge commutes with the supersymmetry generators $q_{a}$ and $\bar{q}_{\dot{a}}$, a BRST exact $U_{a}$ is only possible if the supergravity vertex is also trivial. The light-cone results just discussed can be conveniently illustrated by the diagram depicted in figure 1, which maps the supersymmetric structure of the operators $U_{j, i}, U_{j, \dot{a}}, U_{a, i}$ and $U_{a, \dot{a}}$ before and after the $\ell \rightarrow \infty$ limit, cf. equations (3.28), (4.8) and (4.11).

In terms of the fields introduced in (4.11), the following supersymmetry transformations hold,

$$
\begin{aligned}
\delta \bar{\psi}_{\dot{a}} & =\frac{3 i \sqrt{2}}{64} \partial_{+} A_{i} \sigma_{a \dot{a}}^{i} \epsilon_{a}+\frac{i \sqrt{2}}{384} \partial_{+} A_{i j k} \sigma_{a \dot{a}}^{i j k} \epsilon_{a} \\
\delta A_{i} & =2 i \epsilon_{a} \psi_{a}^{i}-6 i \epsilon_{a} \sigma_{a \dot{a}}^{i} \bar{\psi}_{\dot{a}} \\
\delta \psi_{a}^{i} & =-\frac{7 i \sqrt{2}}{64} \partial_{+} A_{i} \epsilon_{a}+\frac{i \sqrt{2}}{64} \partial_{+} A^{j} \sigma_{a b}^{i j} \epsilon_{b}-\frac{5 i \sqrt{2}}{128} \partial_{+} A_{i j k} \sigma_{a b}^{j k} \epsilon_{b}+\frac{i \sqrt{2}}{128} \partial_{+} A_{j k l} \sigma_{a b}^{i j k l} \epsilon_{b} \\
\delta A_{i j k} & =2 i \epsilon_{a} \sigma_{a \dot{a}}^{i j k} \bar{\psi}_{\dot{a}}-2 i\left[\epsilon_{a} \sigma_{a b}^{i j} \psi_{b}^{k}+\epsilon_{a} \sigma_{a b}^{j k} \psi_{b}^{i}+\epsilon_{a} \sigma_{a b}^{k i} \psi_{b}^{j}\right]
\end{aligned}
$$

where $\epsilon_{a}$ is a constant fermionic parameter.

Naturally, we can try to infer a covariant formulation of the dynamics of these physical degrees of freedom, their equations of motion and gauge transformations. We found that the bosonic sector can be described by two vector fields, $A_{m}$ and $B_{m}$, and a 3 -form, $A_{m n p}$, with equations of motion

$$
\begin{aligned}
\partial_{m} A^{m} & =0 \\
\partial_{m} B^{m} & =0 \\
\partial^{p} A_{m n p} & =\partial_{m} B_{n}-\partial_{n} B_{m} .
\end{aligned}
$$

The fermionic sector is represented by a spinor $\psi^{\alpha}$ and a vector-spinor $\psi_{m \alpha}$ satisfying

$$
\begin{aligned}
\partial_{m}\left(\gamma^{m} \psi\right)_{\alpha} & =0, \\
\partial_{m}\left(\gamma^{m} \psi_{n}\right)^{\alpha} & =0,
\end{aligned}
$$




$$
\begin{aligned}
\left(\gamma^{m} \psi_{m}\right)^{\alpha} & =0 \\
\partial^{m} \psi_{m \alpha} & =0
\end{aligned}
$$

In addition, we have to supplement the above fields with gauge degrees of freedom, which can be summarized by the transformations

$$
\begin{aligned}
\delta A_{m} & =\partial_{m} \Lambda, \\
\delta \psi_{m \alpha} & =\partial_{m} \Lambda_{\alpha}, \\
\delta B_{m} & =\partial_{m} \Omega+\partial^{n} \Omega_{m n}, \\
\delta A_{m n p} & =\partial_{m} \Omega_{n p}+\partial_{n} \Omega_{p m}+\partial_{p} \Omega_{m n},
\end{aligned}
$$

with gauge parameters $\Lambda, \Lambda_{\alpha}, \Omega$ and $\Omega_{m n}$.

It can be shown that the equations of motion (4.14) and gauge transformations (4.15) lead to the light-cone degrees of freedom discussed before. Note in particular that the field $B_{m}$ can be gauged to zero but it is required in the covariant form of the supersymmetry transformations (4.13), given by

$$
\begin{aligned}
\delta \psi^{\alpha}= & -\frac{3 i}{64} \partial_{n} A_{m}\left(\gamma^{m n} \xi\right)^{\alpha}-\frac{i}{384} \partial_{q} A_{m n p}\left(\gamma^{m n p q} \xi\right)^{\alpha}, \\
\delta A_{m}= & 2 i\left(\xi \psi_{m}\right)-6 i\left(\xi \gamma_{m} \psi\right), \\
\delta \psi_{m \alpha}= & \frac{7 i}{64} \partial_{n} A_{m}\left(\gamma^{n} \xi\right)_{\alpha}+\frac{i}{64} \partial^{n} A^{p}\left(\gamma_{m n p} \xi\right)_{\alpha}-\frac{i}{64} \partial_{m} A_{n}\left(\gamma^{n} \xi\right)_{\alpha} \\
& +\frac{5 i}{128} \partial_{q} A_{m n p}\left(\gamma^{n p q} \xi\right)_{\alpha}-\frac{i}{128} \partial^{r} A^{n p q}\left(\gamma_{m n p q r} \xi\right)_{\alpha} \\
& -\frac{i}{128} \partial_{m} A_{n p q}\left(\gamma^{n p q} \xi\right)_{\alpha}-\frac{i}{32} \partial_{m} B_{n}\left(\gamma^{n} \xi\right)_{\alpha}, \\
\delta A_{m n p}= & 2 i\left(\xi \gamma_{m n p} \psi\right)-2 i\left[\left(\xi \gamma_{m n} \psi_{p}\right)+\left(\xi \gamma_{n p} \psi_{m}\right)+\left(\xi \gamma_{p m} \psi_{n}\right)\right], \\
\delta B_{m}= & 2 i\left(\xi \gamma_{m} \psi\right)-2 i\left(\xi \psi_{m}\right) .
\end{aligned}
$$

The supersymmetry algebra closes on-shell up to gauge transformations,

$$
\begin{aligned}
{\left[\delta_{1}, \delta_{2}\right] \psi^{\alpha} } & =\left(\xi_{1} \gamma^{m} \xi_{2}\right) \partial_{m} \psi^{\alpha} \\
{\left[\delta_{1}, \delta_{2}\right] A_{m} } & =\left(\xi_{1} \gamma^{n} \xi_{2}\right) \partial_{n} A_{m}+\text { gauge } \\
{\left[\delta_{1}, \delta_{2}\right] \psi_{m \alpha} } & =\left(\xi_{1} \gamma^{n} \xi_{2}\right) \partial_{n} \psi_{m \alpha}+\text { gauge } \\
{\left[\delta_{1}, \delta_{2}\right] A_{m n p} } & =\left(\xi_{1} \gamma^{q} \xi_{2}\right) \partial_{q} A_{m n p}+\text { gauge } \\
{\left[\delta_{1}, \delta_{2}\right] B_{m} } & =\left(\xi_{1} \gamma^{n} \xi_{2}\right) \partial_{n} B_{m}+\text { gauge }
\end{aligned}
$$

These covariant results might be relevant in trying to demonstrate the equivalence between the two descriptions of the ambitwistor string, with worldsheet supersymmetry or using pure spinors. By formulating a sectorized model of the former, it should be simple to determine its massive cohomology and verify that it resembles the first level of the open superstring. If we assume the two descriptions are indeed equivalent, the cohomology obtained in [2] for the heterotic case is incomplete, since its bosonic content is missing one 
vector field (here denoted by $A_{m}$ ). We hope to address this discrepancy in the future, using the results above as a guiding direction to a more thorough analysis of the heterotic ambitwistor string using the RNS formalism.

In the next section we will summarize our results and open problems, presenting an overview of the connection between the sectorized and the ambitwistor strings.

\section{Concluding remarks}

The sectorized model [7] was proposed as a new interpretation of the modifications introduced by Chandía and Vallilo [5, 6] to the pure spinor version of the ambitwistor string [3]. As such, the role of a dimensionful parameter was never really considered before. In the present work, we focused on the heterotic case to show that the sectorized model indeed accommodates a length parameter. That turned out to be a fortunate choice. Had we decided to analyze the type II case instead, no other states in the cohomology would have been found. The reason is that massive states in the sectorized string are open string like, ${ }^{6}$ therefore incompatible with $\mathcal{N}=2$ supersymmetry. In other words, massive states can appear only in the bosonic and heterotic cases.

We pointed out that the length parameter shares a priori no relation with $\alpha^{\prime}$. The reason is that the ambitwistor string was originally built as an infinite tension string $\left(\alpha^{\prime} \rightarrow\right.$ 0 ) while in the sectorized model it appears as the $\ell \rightarrow \infty$ limit. On the other hand, identifying $\ell^{2} \sim \alpha^{\prime}$ apparently leads to no contradiction, as the opposite limits are taken in different theories.

An interesting property of the sectorized string is made explicit when we write everything down in terms of the tension $\mathcal{T} \sim \ell^{-2}$ (or $\mathcal{T} \sim \frac{1}{\alpha^{\prime}}$ ). In this case, the heterotic models considered here (what we called A and B, cf. section (3), and ambitwistors) can be seen as three different facets of the same underlying theory, corresponding respectively to the $\mathcal{T}<0, \mathcal{T}>0$ and $\mathcal{T}=0$ regimes. If one starts with the $\mathrm{B}$ model $(\mathcal{T}>0)$, the physical spectrum comprises $\mathcal{N}=1$ supergravity and another multiplet corresponding to the first massive level of the open superstring. Next, going to $\mathcal{T}=0$ (ambitwistor string), part of the massive spectrum collapses to a supergravity multiplet while the remaining states constitute a new supersymmetric multiplet that includes the 3-form found by Mason and Skinner [2]. Moving on to the $\mathcal{T}<0$ region (A model), the supergravity sector remains unchanged and the BRST closedness conditions insinuate a tachyonic state mirroring the massive level but forbidden by supersymmetry (note that this is a fundamental feature of the pure spinor formalism). In the absence of supersymmetry there should be $M^{2}<0$ states with otherwise the same quantum numbers as the first massive level of the open string spectrum. A similar feature appeared already in [24], but such states were disregarded as unphysical. In [11] this is further explored in a chiral bosonic model and the cohomology is partially suggested. The bosonic string seems to be the most adequate toy model to understand more fundamental aspects of the sectorized string, including the role of the integrated vertices. This is currently under investigation and new results should be available soon.

\footnotetext{
${ }^{6}$ As pointed out in the text, such states mimic the degrees of freedom of the first massive level of the open (super)string, hence the name.
} 
It is interesting to note that our results share some similarities with the chiral strings discussed by Huang et al. in [12], who focused on the factorization of chiral amplitudes. We believe there are still missing connections between the different chiral strings and the subject deserves further attention, including its relation to double field theory and the results of [25].

Perhaps the most intriguing (missing) piece concerns a practical construction of the integrated vertex operator, since the usual construction involving the $b$ ghost does not render a sensible operator. Recall that for the pure spinor sectorized string, the energymomentum tensor, $T$, and the generalized particle-like Hamiltonian, $\mathcal{H}$, were shown to be BRST exact in [7], with the definition of composite operators $b$ and $\tilde{b}$ satisfying

$$
\begin{aligned}
& \{Q, b\}=\frac{1}{\ell^{2}} T, \\
& \{Q, \tilde{b}\}=\mathcal{H} .
\end{aligned}
$$

Curiously, there does not seem to exist an operator that trivializes $T$ in the $\ell \rightarrow \infty$ limit, which might be related to the absence of the Virasoro constraint in the BRST charge (4.1). Using $b$ and $\tilde{b}$ above, the naive definition of the integrated vertex operator, $V$, would be

$$
V \equiv \int d^{2} z\left\{b_{-1},\left[\tilde{b}_{-1}, U\right]\right\}
$$

where $U$ is the corresponding unintegrated vertex operator and the subscript -1 denotes the Laurent mode of the operators. It is straightforward to show that

$$
[Q, V]=\int d^{2} z\left\{\frac{1}{\ell^{2}} \partial\left[\tilde{b}_{-1}, U\right]-\left[b_{-1},\left[\mathcal{H}_{-1}, U\right]\right]\right\}
$$

The surface contribution can be discarded but the second term is not zero, therefore $V$ is not BRST closed. Furthermore, the integrand in $V$ has the wrong conformal dimensions, $(2,0)$ instead of $(1,1)$. In principle, $V$ can be corrected by introducing an abstract BRST closed operator $\bar{\delta}\left[\mathcal{H}_{-1}\right]$ with conformal dimension $(-1,1)$, satisfying

$$
\bar{\delta}\left[\mathcal{H}_{-1}\right] \cdot\left[\mathcal{H}_{-1}, U\right]=0 .
$$

Such construction is possible so far only in the $\ell \rightarrow \infty$ limit, corresponding to the usual delta function in the ambitwistor string vertices. Note also that equation (5.4) is consistent with the absence of surface terms in the BRST closedness analysis of the integrated vertices proposed in [3]. Without a consistent definition of the vertex operators, it does not seem possible to determine from first principles the interactions of the model. We hope that the reintroduction of the parameter $\ell$ might help clarify this subject, perhaps through an unconventional approach to higher-point amplitudes.

Finally, it would be very interesting to find an $\mathcal{N}=1$ supergravity action which incorporates also the multiplet of the 3 -form. The first step in this direction would be to understand the background coupling semi-classically, as we did in [8] for pure supergravity. We believe that this model is closely related to the conformal supergravity described in [26]. It might be useful also to examine whether the $\mathcal{T} \neq 0$ theory can give us any information 
on the coupling of the massive multiplet. In a recent paper [27], Johansson and Nohle introduced a gauge theory with terms of the form $(D F)^{2}$, which gives rise to conformal supergravity when combined with SYM via double copy. Furthermore, they show that there exists a mass deformation of the form $m^{2} F^{2}$ such that one recovers conformal gravity in the $m \rightarrow 0$ limit and Einstein gravity in the $m \rightarrow \infty$ limit. In this sense, their work can be seen as a field-theoretic description of our results with the identification $m \sim \ell^{-1}$. It would be interesting to investigate whether this correspondence could lead to further insights in both theories.

\section{Acknowledgments}

We would like to thank Henrik Johansson for useful discussions. TA acknowledges financial support from the Knut and Alice Wallenberg Foundation under grant 2015.0083. RLJ would like to thank the Grant Agency of the Czech Republic for financial support under the grant P201/12/G028.

\section{A Useful OPE's}

In this appendix we list the OPE's involving the worldsheet fields in both A and B models of the sectorized heterotic string, as well as those in the ghost sector, which are common for both models. These are useful for the cohomology analysis presented in the main text.

The A model. The OPE's in the A model are essentially the same as the ones given in [7], but now we explicitly include the dimensionful parameter $\ell$. They are given by:

$$
\begin{aligned}
d_{\alpha}(z) d_{\beta}(y) & \sim-\frac{P_{m}^{-} \gamma_{\alpha \beta}^{m}}{(z-y)}, & & P_{m}^{ \pm}(z) P_{n}^{ \pm}(y) \sim \mp \frac{2}{\ell^{2}} \frac{\eta_{m n}}{(z-y)^{2}}, \\
d_{\alpha}(z) P_{m}^{-}(y) & \sim-\frac{2}{\ell^{2}} \frac{\left(\gamma_{m} \partial \theta\right)_{\alpha}}{(z-y)}, & d_{\alpha}(z) \Pi^{m}(y) & \sim \frac{\left(\gamma_{m} \partial \theta\right)_{\alpha}}{(z-y)}, \\
P_{m}^{-}(z) \Pi^{n}(y) & \sim-\frac{\delta_{m}^{n}}{(z-y)^{2}}, & P_{m}^{+}(z) \Pi^{n}(y) & \sim-\frac{\delta_{m}^{n}}{(z-y)^{2}} .
\end{aligned}
$$

The B model. The OPE set for the B model is very similar to the A model and can be cast as:

$$
\begin{array}{rlrl}
\hat{d}_{\alpha}(z) \hat{d}_{\beta}(y) & \sim-\frac{\hat{P}_{m}^{+} \gamma_{\alpha \beta}^{m}}{(z-y)}, & \hat{P}_{m}^{ \pm}(z) \hat{P}_{n}^{ \pm}(y) \sim \mp \frac{2}{\ell^{2}} \frac{\eta_{m n}}{(z-y)^{2}} \\
\hat{d}_{\alpha}(z) \hat{P}_{m}^{+}(y) \sim \frac{2}{\ell^{2}} \frac{\left(\gamma_{m} \partial \theta\right)_{\alpha}}{(z-y)}, & \hat{d}_{\alpha}(z) \Pi^{m}(y) \sim \frac{\left(\gamma_{m} \partial \theta\right)_{\alpha}}{(z-y)}, \\
\hat{P}_{m}^{-}(z) \Pi^{n}(y) & \sim-\frac{\delta_{m}^{n}}{(z-y)^{2}}, & \hat{P}_{m}^{+}(z) \Pi^{n}(y) \sim-\frac{\delta_{m}^{n}}{(z-y)^{2}} .
\end{array}
$$


Ghost sector. Finally, the relevant OPE's in the ghost sector are given by:

$$
\begin{aligned}
\bar{b}(z) \bar{c}(y) & \sim \frac{1}{(z-y)}, & N^{m n}(z) N^{p q}(y) & \sim 6 \frac{\eta^{m[p} \eta^{q] n}}{(z-y)^{2}}+2 \frac{\eta^{m[q} N^{p] n}+\eta^{n[p} N^{q] m}}{(z-y)}, \\
J(z) \lambda^{\alpha}(y) & \sim \frac{\lambda^{\alpha}}{(z-y)}, & T(z) N^{m n}(y) & \sim \frac{N^{m n}}{(z-y)^{2}}+\frac{\partial N^{m n}}{(z-y)}, \\
N^{m n}(z) \lambda^{\alpha}(y) & \sim \frac{1}{2} \frac{\left(\gamma^{m n} \lambda\right)^{\alpha}}{(z-y)}, & T(z) J(y) & \sim \frac{8}{(z-y)^{3}}+\frac{J}{(z-y)^{2}}+\frac{\partial J}{(z-y)}, \\
N^{m n}(z) J(y) & \sim \text { regular, } & J(z) J(y) & \sim-\frac{4}{(z-y)^{2}} .
\end{aligned}
$$

Open Access. This article is distributed under the terms of the Creative Commons Attribution License (CC-BY 4.0), which permits any use, distribution and reproduction in any medium, provided the original author(s) and source are credited.

\section{References}

[1] F. Cachazo, S. He and E.Y. Yuan, Scattering of massless particles in arbitrary dimensions, Phys. Rev. Lett. 113 (2014) 171601 [arXiv:1307.2199] [INSPIRE].

[2] L. Mason and D. Skinner, Ambitwistor strings and the scattering equations, JHEP 07 (2014) 048 [arXiv: 1311.2564] [INSPIRE].

[3] N. Berkovits, Infinite tension limit of the pure spinor superstring, JHEP 03 (2014) 017 [arXiv: 1311.4156] [INSPIRE].

[4] H. Gomez and E.Y. Yuan, $N$-point tree-level scattering amplitude in the new Berkovits' string, JHEP 04 (2014) 046 [arXiv:1312.5485] [INSPIRE].

[5] O. Chandía and B.C. Vallilo, Ambitwistor pure spinor string in a type-II supergravity background, JHEP 06 (2015) 206 [arXiv:1505.05122] [INSPIRE].

[6] O. Chandía and B.C. Vallilo, On-shell type-II supergravity from the ambitwistor pure spinor string, Class. Quant. Grav. 33 (2016) 185003 [arXiv:1511.03329] [INSPIRE].

[7] R.L. Jusinskas, Notes on the ambitwistor pure spinor string, JHEP 05 (2016) 116 [arXiv: 1604.02915] [INSPIRE].

[8] T. Azevedo and R.L. Jusinskas, Background constraints in the infinite tension limit of the heterotic string, JHEP 08 (2016) 133 [arXiv: 1607.06805] [INSPIRE].

[9] N. Berkovits and P.S. Howe, Ten-dimensional supergravity constraints from the pure spinor formalism for the superstring, Nucl. Phys. B 635 (2002) 75 [hep-th/0112160] [InSPIRE].

[10] W. Siegel, Amplitudes for left-handed strings, arXiv:1512.02569 [INSPIRE].

[11] E. Casali and P. Tourkine, On the null origin of the ambitwistor string, JHEP 11 (2016) 036 [arXiv: 1606. 05636] [INSPIRE].

[12] Y.-t. Huang, W. Siegel and E.Y. Yuan, Factorization of chiral string amplitudes, JHEP 09 (2016) 101 [arXiv: 1603.02588] [INSPIRE].

[13] E. Witten, Perturbative gauge theory as a string theory in twistor space, Commun. Math. Phys. 252 (2004) 189 [hep-th/0312171] [INSPIRE]. 
[14] N. Berkovits and E. Witten, Conformal supergravity in twistor-string theory, JHEP 08 (2004) 009 [hep-th/0406051] [INSPIRE].

[15] T. Azevedo and O.T. Engelund, Ambitwistor formulations of $R^{2}$ gravity and $(D F)^{2}$ gauge theories, arXiv:1707.02192 [INSPIRE].

[16] B. Zwiebach, Curvature squared terms and string theories, Phys. Lett. B 156 (1985) 315.

[17] N. Berkovits, Covariant quantization of the superparticle using pure spinors, JHEP 09 (2001) 016 [hep-th/0105050] [INSPIRE].

[18] N. Berkovits and O. Chandía, Massive superstring vertex operator in $D=10$ superspace, JHEP 08 (2002) 040 [hep-th/0204121] [INSPIRE].

[19] R.L. Jusinskas, Spectrum generating algebra for the pure spinor superstring, JHEP 10 (2014) 22 [arXiv: 1406.1902] [INSPIRE].

[20] L. Brink, M.B. Green and J.H. Schwarz, Ten-dimensional supersymmetric Yang-Mills theory with SO(8)-covariant light cone superfields, Nucl. Phys. B 223 (1983) 125 [InSPIRE].

[21] A. Bagchi, R. Gopakumar, I. Mandal and A. Miwa, GCA in 2d, JHEP 08 (2010) 004 [arXiv: 0912.1090] [INSPIRE].

[22] F. Lizzi, B. Rai, G. Sparano and A. Srivastava, Quantization of the null string and absence of critical dimensions, Phys. Lett. B 182 (1986) 326 [INSPIRE].

[23] R.L. Jusinskas, On the field-antifield (a)symmetry of the pure spinor superstring, JHEP 12 (2015) 136 [arXiv:1510.05268] [INSPIRE].

[24] S. Hwang, R. Marnelius and P. Saltsidis, A general BRST approach to string theories with zeta function regularizations, J. Math. Phys. 40 (1999) 4639 [hep-th/9804003] [INSPIRE].

[25] O. Hohm, W. Siegel and B. Zwiebach, Doubled $\alpha^{\prime}$-geometry, JHEP 02 (2014) 065 [arXiv: 1306 .2970] [INSPIRE].

[26] M. de Roo, The $R^{2}$ action in $D=10$ conformal supergravity, Nucl. Phys. B 372 (1992) 243 [INSPIRE].

[27] H. Johansson and J. Nohle, Conformal gravity from gauge theory, arXiv:1707.02965 [INSPIRE]. 